\title{
Multimode Waveguide Van-Atta Array
}

\author{
Y.V. Yukhanov, T.Y. Privalova, I.V. Merglodov, E.V. Kriuk \\ Southern Federal University \\ Russia
}

\begin{abstract}
In this paper, approximate formulas are obtained for the monostatic and bistatic diagrams of radar cross-section of waveguide Van-Atta array considering excitation and propagation of higher types of waves in tracts. Their contribution to the scattered field is studied. The required number of higher types of waves and their propagation conditions are estimated to ensure the widest possible monostatic diagram of radar crosssection. Based on this analysis, the designs of multimode waveguide Van-Atta arrays are offered with tracts, providing non-dispersible transfer of fields between each pair of emitters.
\end{abstract}

Keywords- antenna array; frequency-selective surface; shield; radiation pattern; scattering characteristic

\section{INTRODUCTION}

Since its first appearance in 1959 [1] and till present day Van-Atta arrays are widely used in navigation, communication and radar systems due to its great signal control abilities. Constant increase of practical application of Van-Atta arrays calls for deeper analysis of its propagation and scattering characteristics taking into account the impact of spatial interaction of array elements, appearance of higher types of waves in connecting transmission lines etc..

In papers [2] $\div$ [5] exact and approximate solutions were obtained of two-dimensional problems of diffraction of a plane wave on planar waveguide Van-Atta array, elements of which are separated by ideally conductive or impedance flange (fig. 1). Approximate solution was obtained taking into account the impact of impedance flange on singlemode plumbings connecting the radiators. Results of exact solutions in [2] - [5] show, that by using multimode non-dispersible waveguides monostatic diagram of Radar Cross-Section can be significantly expanded. Though it was not clear how to practically implement the transfer of all higher types of waves with equal phase velocity through plumbings connecting the radiators.

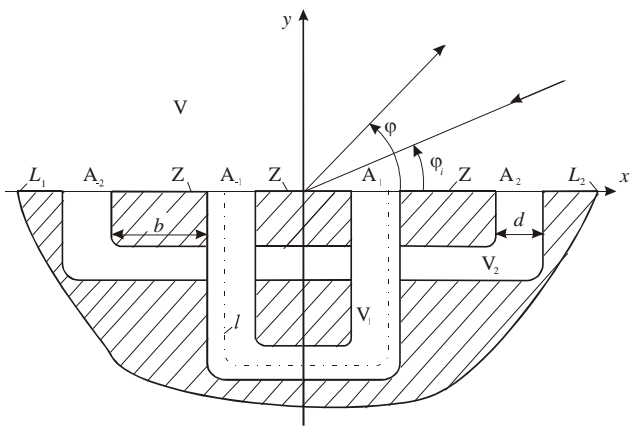

FIGURE I. TWO-DIMENSIONAL MODEL OF VAN-ATTA ARRAY.

The purpose of this work is to achieve maximum possible expansion of working angular sector of Van-Atta array, based on multimode waveguides. To obtain approximate formulas for monostatic diagram of radar cross-section of waveguide Van-Atta array taking into account the excitation and propagation of higher types of waves in connecting transmission lines. To study their contribution to the scattered field. To show the possibility of implementing multimode in three-dimensional models of AA structures. Conduct an analysis of the scattering characteristics of two- and threedimensional models of antennas.

\section{MUltimode VAN-ATTA ARRAY}

Suppose that elements of Van-Atta array (fig. 1), located on the perfectly conducting plane and consisting of $N$ pairs of identical radiators in the form of planar waveguides with aperture sizes $d>\lambda$, in transmission lines in the form of parallel plate waveguides $V_{n}(n=1 \ldots N)$ of equal length $l$ there can be propagated $N_{T}$ waves.

Let us consider the approximate solution. At the same time we ignore the interaction of the electromagnetic field between the apertures $A_{ \pm M}$ through the upper halfspace $y>0$ (fig. 1). The electric field vectors in the apertures $A_{ \pm M}$ of coupled waveguides can be found from the following system of Fredholm equations of the 1 st kind:

$$
\begin{aligned}
& \int_{A_{M}} E_{x}^{\langle M\rangle}\left(x^{\prime}\right)\left[H_{z}^{M\langle M\rangle}\left(x, x^{\prime}\right)+H_{z}^{\prime \prime}\left(x, x^{\prime}\right)\right] d x^{\prime}+ \\
& +\int_{A_{-M}} E_{x}^{\langle-M\rangle}\left(x^{\prime}\right) H_{z}^{M\langle-M\rangle}\left(x, x^{\prime}\right) d x^{\prime}=-2 e^{i k x \cos \varphi_{i}}, x \in A_{M} ; \\
& \int_{A_{-M}} E_{x}^{\langle-M\rangle}\left(x^{\prime}\right)\left[H_{z}^{M\langle M\rangle}\left(x, x^{\prime}\right)+H_{z}^{\prime \prime}\left(x, x^{\prime}\right)\right] d x^{\prime}+ \\
& +\int_{A_{M}} E_{x}^{\langle M\rangle}\left(x^{\prime}\right) H_{z}^{M\langle-M\rangle}\left(x, x^{\prime}\right) d x^{\prime}=-2 e^{i k x \cos \varphi_{i}}, x \in A_{-M}, \\
& \operatorname{where}_{z}^{M\langle M\rangle}\left(x, x^{\prime},\right)=-\frac{k}{d} \sum_{n=0}^{\infty} \frac{\varepsilon_{n}}{i k_{n} \operatorname{tg} k_{n} l} \cos \gamma_{n} x^{\prime} \cos \gamma_{n} x ; \\
& H_{z}^{M\langle-M\rangle}\left(x, x^{\prime},\right)=-\frac{k}{d} \sum_{n=0}^{\infty} \varepsilon_{n} \frac{\varepsilon_{n}}{i k_{n} \sin k_{n} l} \cos \gamma_{n} x^{\prime} \cos \gamma_{n} x ; \\
& H_{z}^{M}\left(x, x^{\prime}\right)=-\frac{k}{2} H_{0}^{2}\left(k\left|x-x^{\prime}\right|\right) ; \quad \gamma_{n}=\frac{n \pi}{d} .
\end{aligned}
$$

For monostatic diagrams of radar cross-section we obtain an equation [5]: 


$$
\begin{gathered}
F\left(\varphi_{i}\right)=F_{A R}\left(\varphi_{i}\right)+F_{f}\left(\varphi_{i}\right), \\
F_{A R}\left(\varphi_{i}\right)=k d N e^{-i k d \cos \varphi_{i}}\left(\frac{1+\sin \varphi_{i}}{2}\right)^{2} \sum_{n=0}^{\infty} \varepsilon_{n} \frac{k_{n}}{k}\left|f_{n}\left(\varphi_{i}\right)\right|^{2} e^{-i k_{n} l} ;(3) \\
F_{f}\left(\varphi_{i}\right)=\sin \varphi_{i} \frac{\sin \left[2 k L \cos \varphi_{i}\right]}{2 \cos \varphi_{i}}-\frac{k d}{2} e^{-i k d \cos \varphi_{i}} \frac{\sin \left[k h N \cos \varphi_{i}\right]_{*}}{\sin \left[k h \cos \varphi_{i}\right]} \\
*\left(\sum_{n=0}^{\infty} \varepsilon_{n} \frac{k_{n}}{k}\left(e^{-i k h N \cos \varphi_{i}}\left(f_{n}^{*}\left(\varphi_{i}\right)\right)^{2}+e^{i k h N \cos \varphi_{i}}\left(f_{n}\left(\varphi_{i}\right)\right)^{2}\right)\right), \\
\text { where } f_{n}\left(\varphi_{i}\right)=\frac{1}{d} \int_{0}^{d} e^{i k x \cos \varphi_{i}} \cos \gamma_{n} x d x .
\end{gathered}
$$

Since each mode propagates in the waveguide with its own speed $v_{n}=c / \sqrt{k^{2}-\gamma_{n}^{2}}$ ( $c$ - speed of light), then in the process of propagation through the waveguide $V_{M}(M=1 \ldots N)$ incident waves front is significantly distorted. To invert it $\left(e^{i k x \cos \varphi_{i}}\right)$ without distortions $\left(e^{-i k x \cos \varphi_{i}}\right)$ it is necessary to ensure the non-dispersible distribution of all types of waves.

Suppose, for example $k_{n}=k\left(v_{n}=c\right)$, then equations (1)-(4) will take the form:

$$
\begin{gathered}
E_{x}^{\langle M\rangle}(x)=e^{i k\left(x_{M}-d / 2\right) \cos \varphi_{i}} e^{i k x \cos \varphi_{i}}-e^{-i k\left(x_{M}-d / 2\right) \cos \varphi_{i}} e^{-i k l} e^{-i k x \cos \varphi_{i}} \\
E_{x}^{\langle-M\rangle}(x)=-e^{i k\left(x_{M}-d / 2\right) \cos \varphi_{i}} e^{-i k l} e^{i k \cos \varphi_{i}}+e^{-i k\left(x_{M}-d / 2\right) \cos \varphi_{i}} e^{-i k \cos \varphi_{i}} \\
F_{A R}\left(\varphi_{i}\right)=k d N\left(\frac{1+\sin \varphi_{i}}{2}\right)^{2} e^{-i k l} ; \\
F_{f}\left(\varphi_{i}\right)=\sin \varphi_{i} \frac{\sin \left[2 k L \cos \varphi_{i}\right]}{2 \cos \varphi_{i}}- \\
-\cos \left(k h N \cos \varphi_{i}\right) \frac{\sin \left[k d \cos \varphi_{i}\right]}{\cos \varphi_{i}} \frac{\sin \left[k h N \cos \varphi_{\dot{i}}\right]}{\sin \left[k h \cos \varphi_{i}\right]}
\end{gathered}
$$

Thus, as expected, non-dispersible transmission lines in a multimode mode during signal transmission from the receiving aperture to the emitting perform (the formula (5) and (6)) the inverting of the incident waves front without distortions. This, in turn, provides such Van-Atta array with widest possible monostatic diagram of radar cross-section (10).

As an example, fig. 2 shows $\mathrm{RP}$ with parameters $d=4 \lambda ; b=0$

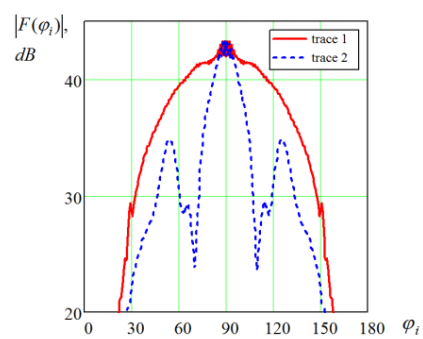

FIGURE II. DIAGRAMS OF RADAR CROSS-SECTION OF MULTIMODE VAN-ATTA ARRAY. $d=4 \lambda ; b=0 ; l=10.5 \lambda ; N=5$ for non-dispersible (curve 1) and dispersible (curve 2) transmission lines.

As we can see, multimode array with non-dispersible lines has significantly better diagram. To ensure the same level of reflected signal in the single-mode array ten times more radiators with $d=0.4 \lambda$ will be required.

It remains to figure out how to practically implement the transfer of all higher types of waves via tracts connecting the emitters with the same phase velocity.

\section{3D MODEL OF MULTIMODE VAN-ATTA ARRAY}

Below there have been made an attempt to combine the advantages of multimode waveguides and singlemode transmission lines connected between them. Research was conducted in Ansoft HFSS software package for threedimensional models of Van-Atta arrays with singlemode and multimode waveguide radiators.

On the example of a rectangular waveguide with $23 \times 69 \mathrm{~mm}$ cross section and $100 \mathrm{~mm}$ length it is shown (fig. 3 ), that the incident at an angle of 450 plane electromagnetic wave $(\mathrm{f}=10.5 \mathrm{GHz})$, passing through it, almost completely destroys its phase structure.

If we connect multimode waveguides with some of the singlemode waveguides at the distance from the apertures at which the phase of the received signal still remains linear, it is possible to transmit the signal without significant phase distortions over the entire length of the waveguide into reradiating aperture.

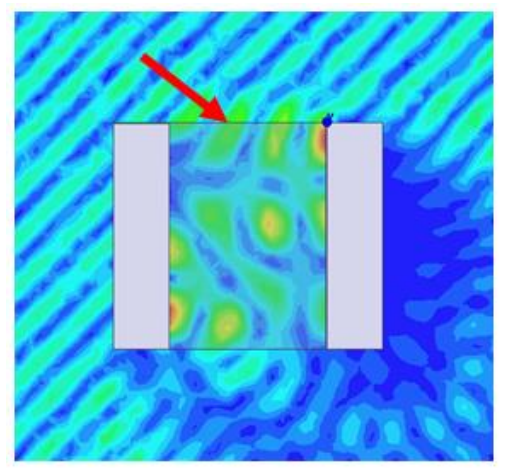

FIGURE III. STRUCTURE OF THE WAVE IN THE WAVEGUIDE.

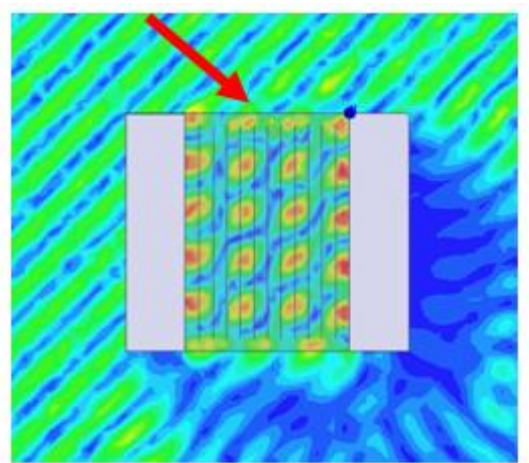

FIGURE IV. STRUCTURE OF THE WAVE IN THE WAVEGUIDE. 
Figure 4 shows a picture of the field transmitted through the $23 \times 69 \mathrm{~mm}$ waveguide with length of $100 \mathrm{~mm}$, which at $10 \mathrm{~mm}$ distance from apertures goes into 12 waveguides with $23 \times 5.75 \mathrm{~mm}$ cross-section.

As we can see, in this design the phase of the received field with virtually no distortion is transmitted to the lower aperture. Thus, similar design can be used in creation of Van-Atta arrays.

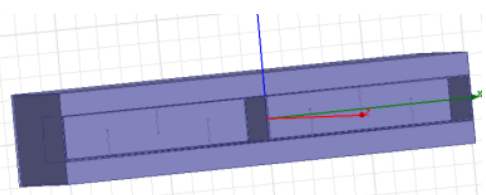

a) a pair of multimode waveguides in a multimode mode

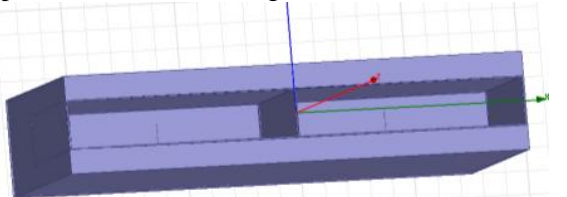

b) a pair of multimode waveguides in a singlemode mode

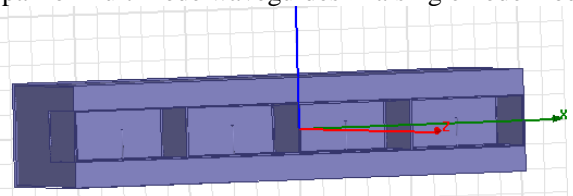

c) two pairs of singlemode waveguides in a singlemode mode

FIGURE V. WAVEGUIDE VAN-ATTA ARRAYS.

Consider the implementing of a multimode waveguide Van-Atta array with the example of one pair of waveguide radiators with aperture size of 50x10 mm (fig. 5,a). The waveguides are closely adjacent to each other, they are separated by a wall with thickness of $1 \mathrm{~mm}$. In such waveguides three types of waves $\mathrm{H} 10, \mathrm{H} 20$ and $\mathrm{H} 30$ can be applied. As a non-dispersible transmission lines three coaxial lines located inside a metal box surrounding the waveguide are selected.

For comparison, the pair of multimode waveguides in single-mode case, connected by a single coaxial line (fig. 5,b) and two pairs of single-mode waveguides of $25 \times 10 \mathrm{~mm}$ (fig. 5), each of which is connected to its coaxial line are considered.

In fig. 6,a-c the monostatic diagrams of radar cross-section in the plane $\mathrm{H}$ for Van-Atta arrays, shown in fig. 5, a (curves 1), fig. 5,b (curves 2) and fig. 5,c (curves 3 ) with the frequency of $10.5 \mathrm{GHz}$ (fig.6,a), $10.8 \mathrm{GHz}$ (fig. 6,b) and $11.0 \mathrm{GHz}$ (fig. 6,c) are given.

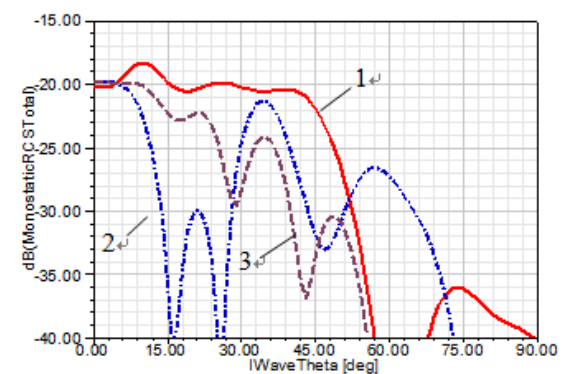

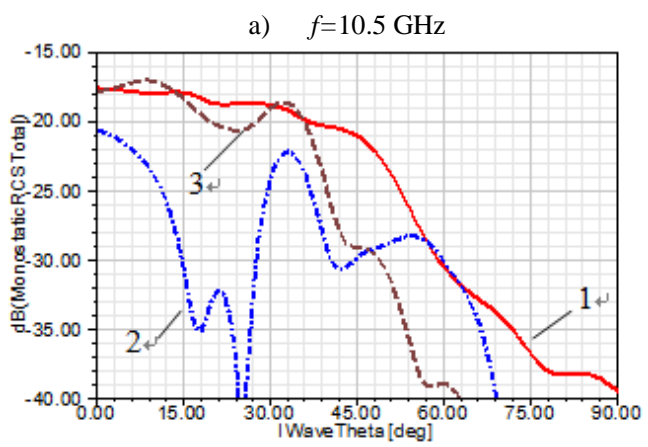

b) $f=10.8 \mathrm{GHz}$

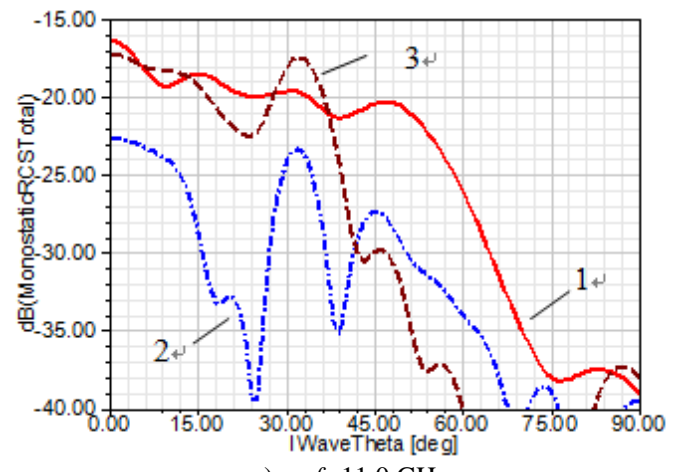

c) $f=11.0 \mathrm{GHz}$

FIGURE VI. MONOSTATIC DIAGRAMS OF RADAR CROSS-SECTION.

As we can see, even such simple design of one pair of three-mode waveguide radiators allows to implement the most extensive (among the considered constructions of Van-Atta arrays with the equal size apertures (fig.5)) monostatic diagrams of radar cross-section with sector angles of \pm 450 for $-3 \mathrm{~dB}$ level.

\section{CONCLUSION}

Obtained approximate formulas for monostatic diagram of radar cross-section waveguide Van-Atta array, taking into account the excitation and propagation of both main and higher types of waves in tracts connecting the emitters. Their contribution to the scattered field is studied. Conditions to ensure the widest possible (8) monostatic diagram of radar cross-section are obtained. On an example of calculating the scattering characteristics of the array in the Ansoft HFSS software package the possibility is shown of implementing the two-element Van-Atta array based on three-mode waveguides connected by three coaxial lines with working sector of angles of \pm 450 for $-3 \mathrm{~dB}$ level.

\section{ACKNOWLEDGMENTS}

This work was performed as project part of the state task No. 8.2461.2014/K of the Ministry of Education of Russia.

\section{REFERENCES}

[1] Van Atta L.C., "Electromagnetic Reflector", USPatent No. 2908002, Oct. 1959.

[2] Privalova T. Yu., Yukhanov Yu.V. "Plane wave scattering on the twodimensional model of VanAtta array”//Antennas, 2007. №5(120), pp.2328.

[3] Privalova T. Yu., Sinyavsky G.P., Yukhanov Yu.V. "Scattering characteristics analysis of two-dimensional Van-Atta 
array"//Electromagnetic waves and electronic systems, 2007, Vol.12, №5, pp.58-65.

[4] Privalova, T.Y.; Yukhanov, A.Y.; Yukhanov, Y.V. Plane wave scattering on Van-Atta electromagnetic reflector// Electromagnetics in Advanced Applications (ICEAA), 2011 International Conference on DOI: 10.1109/ICEAA.2011.6046309, Page(s): $347-350$.

[5] Privalova, T.Y. ; Yukhanov, A.Y. ; Merglodov, I.V. Scattering characteristics of multimode waveguide van-atta array//Electromagnetics in Advanced Applications (ICEAA), 2014 International Conference on DOI: 10.1109/ICEAA.2014.6903867, Page(s): 317 - 320 . 